\title{
STELLAR WINDS AND CORONAE IN COOL STARS
}

\author{
A.K. Dupree and L. Hartmann \\ Harvard-Smithsonian Center for Astrophysics \\ Cambridge, MA 02138/USA
}

\section{ABSTRACT}

Recent observational and theoretical results are reviewed that pertain to the presence and characteristics of stellar coronae and winds in latetype stars. It is found that stars - principally dwarfs - exist with "hot" coronae similar to the Sun with thermally driven winds. For stars. at the lowest effective temperatures, and gravities characteristic of supergiant and giant stars, high temperature $\left(\sim 10^{5} \mathrm{~K}\right)$ atmospheres are absent (or if present are substantially weaker than in the dwarf stars), and massive winds are present. There also exist "hybrid" examples - lumi nous stars possessing both a "hot" corona and a supersonic stellar wind. Constraints for theoretical models are discussed.

\section{INTRODUCTION}

The study of stellar chromospheres, coronae, and winds has received new impetus from recent ultraviolet and $X$-ray observations. A general outline of the presence and behavior of chromospheres and coronae in latetype stars is beginning to emerge from this new data. In this review, we emphasize the results of the ultraviolet observations, and make comparisons with existing theory. The $X$-ray data are more preliminary so these are considered briefly. Finally, we treat mass loss in the latetype stars, concentrating particularly on the relationship of gas temperature to wind structure.

Optical spectra of most cool stars ( $\mathrm{T}_{\text {eff }} \leqslant 6500 \mathrm{~K}$ ) present an emission core in the Ca II ( $\lambda 3934, \lambda 3968)$ lines which has long signaled the presence of an atmospheric region hotter than the stellar photosphere, and by analogy with the Sun, called a chromosphere. The first ultraviolet measurements were principally of the strong resonance lines, Mg II, O I: and $C$ II which gave evidence for additional chromospheric material at temperatures up to $40,000 \mathrm{~K}$. In two stars - Procyon ( $\alpha$ CMi) and Capella (Alpha Aur) - species at temperatures $23 \times 10^{5} \mathrm{~K}$ were detected (Dupree 1975; Evans, Jordan, and Wilson 1975; Vitz et al. 1976). X-ray measurements, sensitive enough to detect single "normal" stars or we11-separated binaries were sparse, but a few sources-Capella (G5 III + GO III), Eta Bootis (GO IV), Vega (A0 V), Alpha Cen A (G2 V)-had been detected in the 
soft X-ray band $\sim 0.2-1 \mathrm{keV}$ (Catura, Acton, and Johnson 1975; Mewe et a1. 1975; Topka et al. 1979; Nugent and Garmire 1978). From this meager sampling, it was possible to say that stars had chromospheres and coronae - perhaps similar in structure to the Sun in the case of Procyon (Evans, Jordan, and Wilson 1975) but more like a solar active region in Capella (Dupree 1975).

That substantial mass loss occurs in luminous cool stars has been known for quite some time (Deutsch 1956, 1960) from optical work. More recent observations in UV lines have expanded knowledge of mass loss, including cases where optical circumstellar lines are not seen (e.g. Dupree 1976). The detection of circumstellar lines of low excitation ions clearly showed that such winds are not coronal - that is, driven by thermal expansion but must be accelerated by some other process (Weymann 1962). Since dwarfs are presumed to have solar-type coronal mass loss, a transition between the two extreme cases - hot coronae and cool winds - must occur somewhere in the H-R diagram. Reimers (1977) suggested that the disappearance of optical circumstellar lines below an observational line in the H-R diagram was related to the increasing ionization in the circumstellar envelope.

It is clear that a knowledge of the temperature structure of late-type stellar atmospheres and envelopes is extremely important in understanding the nature of mass ejection. Such information is not readily derivable from optical data. With the advent of UV and X-ray measurements, which probe high temperature material, it is now possible to discern the general outline of the presence or absence of hot gas and its correspondence with mass loss. Such an understanding is preliminary, because of incompleteness of data and also because of observational selection effects, but our knowledge of stellar coronae and winds has advanced markedly in the last year or two.

\section{ULTRAVIOLET OBSERVATIONS}

\section{a) Dwarf Stars}

U1traviolet spectra of late-type "quiet" dwarf stars appear generally similar to that of the Sun in the presence of lines typical of the solar transition region and corona (see for instance Brown, Jordan, and Wilson 1979; Dupree et al. 1979; Dupree 1980; Linsky and Haisch 1979; Linsky et a1. 1978). The line ratios and surface fluxes are usually within a factor of 2 or 3 of solar values. 
The active dwarfs defined as those with strong Ca II emission, and flare stars show a similar spectrum; however the surface flux of the lines is larger than solar values, by factors of 4 to 20 and is remarkably independent of photospheric temperature (Hartmann et al. 1979a). The single dwarf stars confirm the presence of chromospheres, transition regions, and coronae that are similar to the solar atmosphere although the active stars have radiative losses at a rate comparable to those found in solar active regions.

\section{b) Giant Stars}

In the more luminous stars the character of the atmosphere appears to change more dramatically from the solar prototype. Figure 1 illustrates typical spectra of single giant stars. It is important to notice that the signal-to-noise ratio is not optimum in many of these spectra and

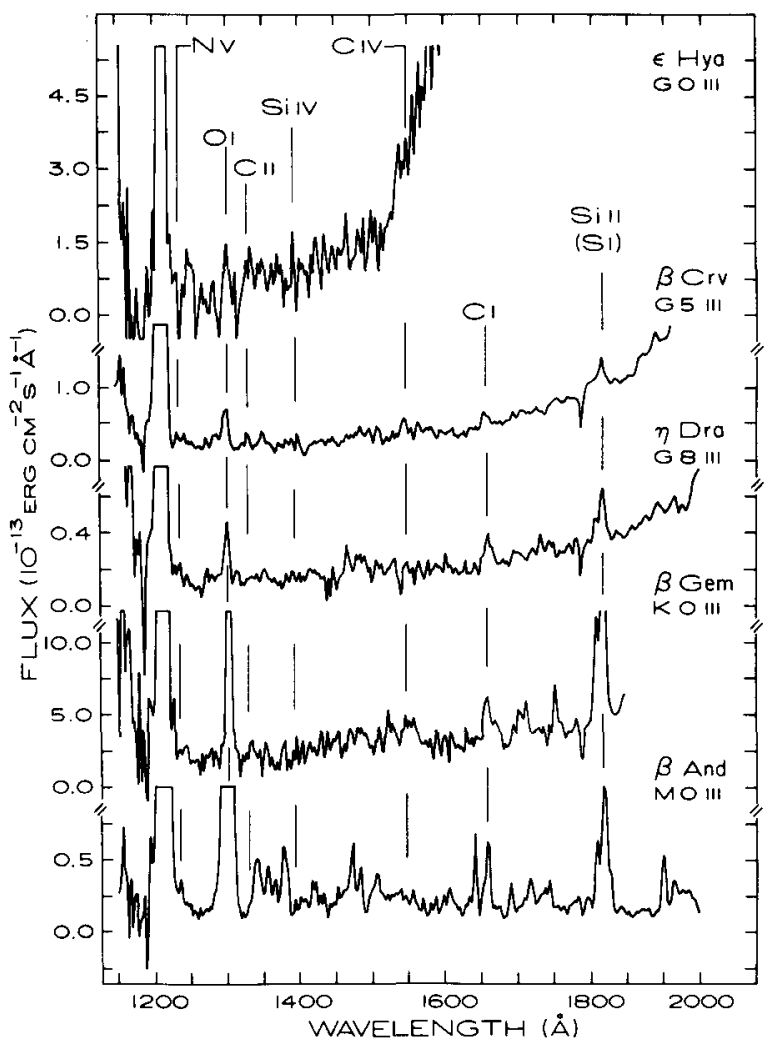

Figure 1 - IUE spectra of selected single giant stars arranged in order of decreasing effective temperature. Note the strong $O I_{\text {and }} \mathrm{Si} I \mathrm{I} I$ ines and the apparent presence of $\mathrm{C}$ IV in $\beta \mathrm{CrV}$ and $\beta$ Gem. Geocoronal Ly- $\alpha$ has been truncated in this figure as has $0 \mathrm{I}$ in $\beta$ Gem and $\beta$ And. 
that an inference concerning the presence or absence of $C$ IV as an example, is particularly difficult for many stars. The spectra are dominated by the lines of $\mathrm{Si} I \mathrm{I}$ and $\mathrm{C} I$, all species formed at $\mathrm{T}_{\mathrm{e}} \sim 10^{4} \mathrm{~K}$. The strong $O I$ multiplet at $\lambda 1302$ is also indicative of these temperatures if the line formation is collisionally controlled; however, in the luminous cool stars this line may be enhanced through radiative excitation by Ly- $\beta$. High temperature species as indicated by C IV ( $\lambda 1550)$ appear to be present in Beta Crv (G5 III) (based on 3 exposures) and in two exposures of Beta Gem (GO III) (see also Carpenter and wing 1979) a1though Eta Dra (G8 III) does not show C IV - again based on multiple exposures. The character of the spectrum changes quite dramatically with $\beta$ And (MO III), with only low temperature lines present. Identifications are difficult at low dispersion. However it is clear in this star that lines of $\mathrm{Fe} I I$ and $S$ I are present, while $N \mathrm{~V}, \mathrm{C} I V$, and $\mathrm{Si}$ IV are not apparent.

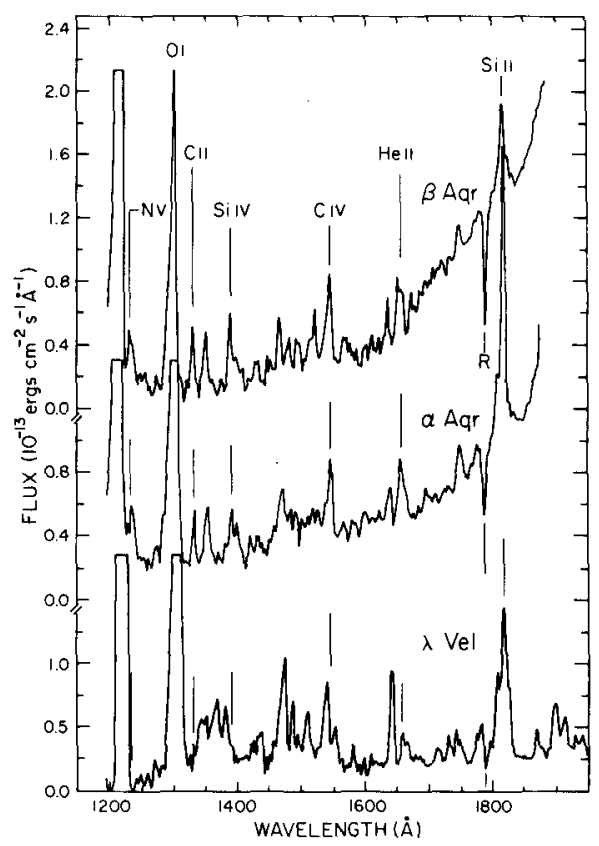

Figure 2 - IUE spectra of 3 supergiant stars: $\beta$ Aqr (GO Ib); $\alpha$ Aqr (G2 Ib); and Vel (K5 Ib) showing low excitation lines in all and the presence of high temperature species $C$ IV and $N V$ in $\alpha$ and $\beta$ Aqr only (From Hartmann, Dupree, and Raymond 1979b). 


\section{c) Supergiant Stars}

IUE spectra of 3 supergiant stars (Fig. 2) show the dominance of $O$ I and Si II as noted in the giant stars, additionally probably fluorescently excited lines of low excitation species C I, S I, Fe II near $\lambda 1450$ and most importantly the clear presence of $C$ IV and $N V$ in the spectra of $\alpha$ and $\beta$ Aqr (Hartmann, Dupree, and Raymond 1979b). The spectrum of $\lambda$ Vel in fact is very similar to that of $\beta$ And. The supergiant $\alpha$ ori (M2 I) also shows no evidence of high temperature material (see for instance Linsky and Haisch 1979) but its spectrum is substantially different from $\lambda$ Vel. The stellar surface fluxes of high temperature lines are approximately equal to 3 or 4 times the solar surface flux in $\alpha$ and $\beta$ Aqr but lower by a factor of $\sim 100$ for $\lambda$ Ve1 (Hartmann, Dupree, and Raymond 1979b; Dupree et a1. 1979).

The chromospheric lines of Mg II (see Fig. 3) for these stars show the blue-red asymmetry that has been associated with a differential chromospheric expansion. However both $\beta$ and $\alpha$ Aqr show also the presence of narrow absorption components in the cores and wings of the lines that correspond to supersonic velocities up to $\sim 125 \mathrm{~km} \mathrm{~s}^{-1}$. These features are found in the $\mathrm{Ca} \mathrm{K}$ profiles as well (Hartmann et a1. 1979b). Thus in $\alpha$ and $\beta$ Aqr we find a corona coexisting with a massive stellar wind.

These data are remarkable for demonstrating that high-temperature gas is not inconsistent with substantial mass loss. In addition, some of the "cool" chromosphere lines in $\lambda$ Vel are present in these G supergiants as well, clearly showing the hybrid nature of the spectrum. The terminal velocity of the shells at $\sim-100 \mathrm{~km} \mathrm{~s}^{-1}$ is also significant in that they are intermediate between solar wind velocities and typical cool supergiant velocities $\sim-20 \mathrm{~km} \mathrm{~s}^{-1}$. Thus these objects may well represent the transition between solar-type winds and cool outflow.

\section{OVERALL PRESENCE OF HOT PLASMA}

The C IV doublet ( $\lambda 1550$ ) can be used to indicate hot plasma with temperatures $2 \times 10^{5} \mathrm{~K}$ - and by analogy with the Sun - we infer the presence of a transition region or corona in these stars. Figure 4 contains a compilation of results from many IUE spectra (Brown, Jordan, and Wilson 1979; Carpenter and Wing 1979; Dupree et a1. 1979; Hartmann et al. 1979 a, b; Linsky and Haisch 1979; Wing 1978). It is apparent that the dwarf stars (luminosity class V) consistently show evidence for C IV, but the more luminous stars (class I and II) show more varied behavior. The coolest stars (spectral type $M$ and later) give no evidence of $C$ IV as 


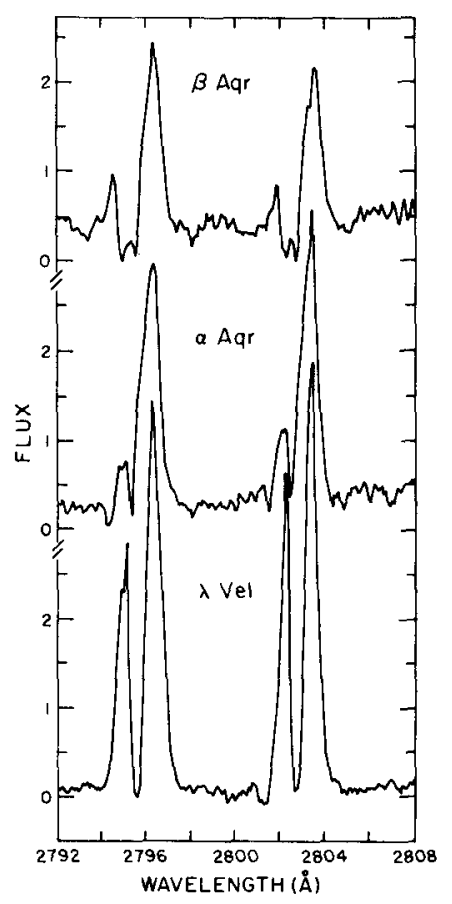

Figure 3 - Strongly asymmetric Mg II emission in 3 supergiant stars (Hartmann, Dupree, and Raymond 1979b). Note that the narrow absorption features in the core and blue wing of the lines of $\alpha$ and $\beta$ Aqr are not as prominent in the spectrum of $\lambda \mathrm{Vel}$.

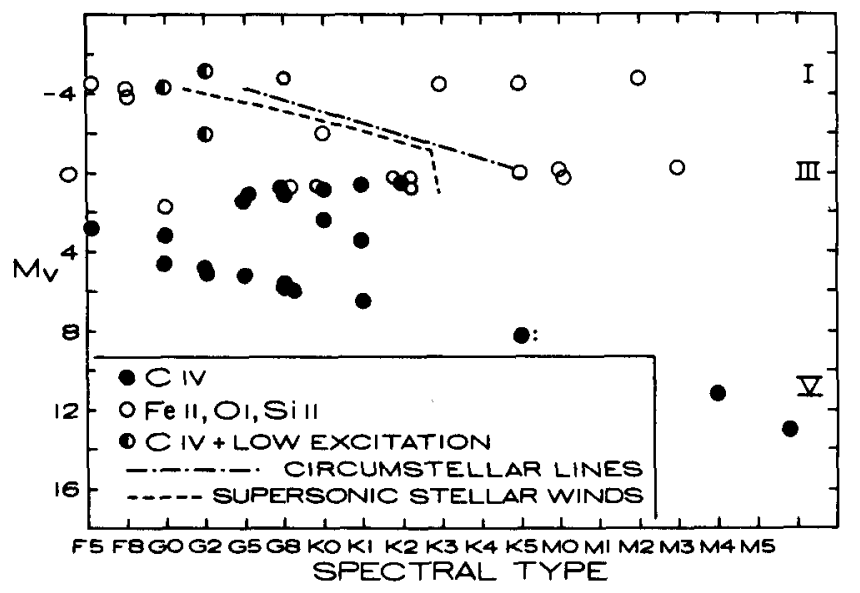

Figure 4 - The presence of emission from C IV and/or cool species in IUE spectra of late-type stars. The broken line corresponds to Mullan's (1978) position of enhanced mass loss. The dot-dash lines denote Reimers (1977) boundary for circumstellar lines. See discussion in text regarding selection effects and uncertainties in detection. 
Wing (1978) first noted for $\gamma$ Cru (M3.4 IIIb). At $G$ and $K$ spectral types there is not a clear change with effective temperature. Along the giant branch, both types occur at spectral type 68 , for instance; in the supergiant stars we find the so-called hybrid atmosphere discussed earlier. The stars showing both C IV and low excitation species lie near the onset of the appearance of circumstellar lines (Reimers 1977 ) and near the "supersonic transition locus" proposed by Mullan (1978) that we discuss later.

Linsky and Haisch (1979) suggested that there were two types of atmospheres - solar and non-solar, that the division was sharp between these types at spectral type KO III, and they speculated that the presence of a massive stellar wind would suppress a corona and eliminate high temperature species. The addition of more data shows that the situation is more complicated. The "division" between hot and cool atmospheres is not at all sharp. Cool atmospheres dominate at a later spectral type than shown by Linsky and Haisch, and hybrid atmospheres exist that exhibit both high temperature lines and substantial mass loss. There is other, more subtle behavior in the line ratios of C II, C IV, and $O I$ emphasized by Brown, Jordan, and Wilson (1979) that support a smooth change in atmospheric structure with decreasing effective temperature. Generally speaking however, stars with large mass loss rates show the coolest emission features and apparently have weak or nonexistent coronae.

These differences of interpretation emphasize the problem of selection effects with small samples of stars. Observers have focussed on bright stars that in many cases possess active chromospheres; additionally, binary systems show enhanced fluxes that may be attributable to rotation. It is not easy to detect C IV in many stars. Many observations do not have sufficient signal-to-noise ratios. Spectra of stars near spectral type G0 III and earlier suffer from direct continuum emission and scattered light in the IUE instrument at short wavelengths. We cannot be sure that the open circles in Fig. 4 are stars without $10^{5} \mathrm{~K}$ gas; we can merely place upper limits on its presence.

We can infer the presence of still hotter material at T $10^{6} \mathrm{~K}$ - from the flux in the He II, $\lambda 1640$ transition. In solar active regions this transition can be enhanced by recombination following photoionization (Raymond, Noyes, and Stopa 1979). In active dwarf stars the line flux confirms the direct measurement of soft X-rays (Hartmann et al. 1979a). The presence of the $\lambda 1640$ transition in the supergiant spectra suggests that a hot 
corona may well exist on these stars; detection could be difficult if soft $X$-ray emission is absorbed by a substantial extended atmosphere. Whereas dwarf stars show He II $\lambda 1640$ (Dupree et al. 1979, Hartmann et al. 1979a), it is interesting that the spectrum of the T Tauri star RU Lupi has very weak, if any $\lambda 1640$ emission, (Gahm et a1. 1979) suggesting that relatively little coronal material is present.

$X-r a y$ measurements from the Einstein Observatory (HEAO-2) could of course give direct information on the highest temperature plasma in these stars, but the data are in a early stage of assessment. The data are consistent with the picture suggested by the ultraviolet results. It is not yet known whether cool supergiants have substantial coronae; the upper limits on coronal emission from very luminous stars can be lowered when exposure times are longer (Rosner 1979). At present, many of the objects in the stellar surveys are faint and have not been well studied optically (Vaiana et al. 1979). Their luminosity classes, activity, or possible binary nature are not known in most cases. The $X$-ray observations should shortly provide important advances in understanding the high temperature plasma in the atmospheres of cool stars.

These observations are beginning to provide some constraints for theories of coronal heating and structure. The radiative losses in a wide variety of single stars vary by a factor of 10 in Mg II (Basri and Linsky 1979) and by a factor of about 100 in higher temperature lines. The ultraviolet emission is independent of stellar effective temperature for active dwarfs, and in general appears to be more dependent on $10 \mathrm{~g} g$ than on $\mathrm{T}_{\mathrm{e}}$. And, in several cases, stars of the same spectral type and gravity exhibit quite different ultraviolet surface fluxes. To date no single theory appears to be successful at reproducing these results.

\section{MASS LOSS}

Line profiles in the optical and ultraviolet have been used to determine the onset and presence of mass loss among late-type stars. Reimers (1977) investigated circumstellar lines in the optical spectra to define aboundary in the HR diagram (see Fig. 5). Additionally blue and red $\mathrm{Mg} I \mathrm{I}$ and Ca II asymmetries occur systematically over a large region (Stencel 1978; Mullan and Stencel 1979). The locus of the absence or weakening of C IV in the coolest stars as taken from Fig. 4 is also shown in Fig. 5.

In the coolest stars substantial mass loss occurs, and these regions correspond to a weakening of the C IV line, and by inference, a corona. 


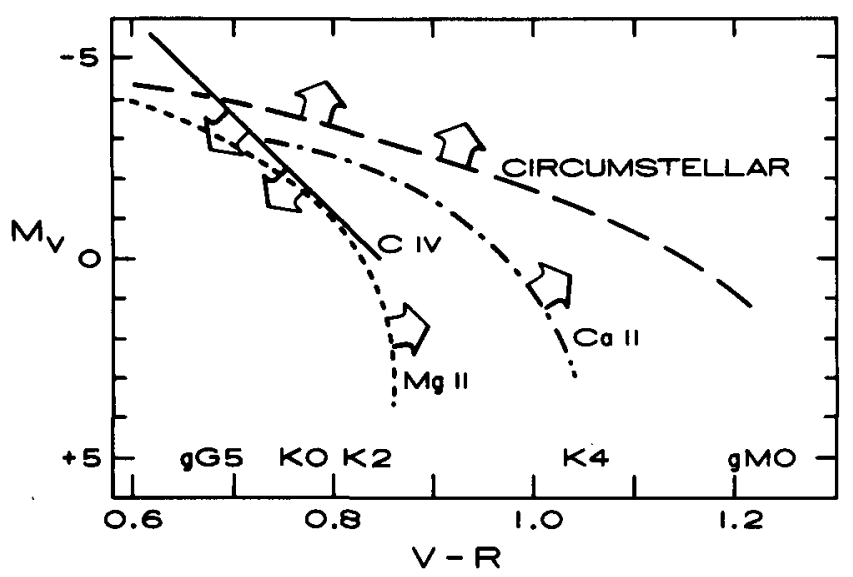

Figure 5 - The appearance of various spectral features as a function of color and luminosity. C IV emission is prominent to the left of the solid line; the ratio of red:blue emission peaks for $\mathrm{Mg}$ II and Ca II is greater than 1 to the right of the appropriate broken 1ines (Stencel 1978; Mullan and Stencel 1979). Circumstellar features are found above the long broken line (Reimers 1977).

There is not a complete anticorrelation of mass loss with high temperature gas however, as shown by the observations of the supergiants $\alpha$ and $B$ Aqr. While the interpretation of $\mathrm{Ca}$ II and Mg II asymmetries is open to argument, this figure suggests a gradual increase in mass loss towards lower effective temperature, with the detection of mass loss in the weakest lines occurring only for the largest mass loss rates.

To date, the most popular theory of mass loss for luminous cool latetype stars is that of radiation pressure on dust (Gehrz and Woolf 1971; Kwok 1975). However, it seems very unlikely that dust forms in the vast majority of late-type stars, so that it is clearly not a universal mechanism. The original work of Durney (1973) has been extended by Mullan (1978) who recently introduced the concept of the "supersonic transition locus", where the sonic points of isothermal coronae are supposed to penetrate down to the top of the chromosphere, resulting in an abrupt enhancement of mass loss from the action of spicules ejecting matter. The semi-empirical calculations assume that Hearn's (1975) minimum-flux corona theory is applicable. The concept of a minimum flux corona is very controversial (see the review by Cassinelli 1979), and the calculation itself is based on a scaling of empirical pressures that is now known to be uncertain (Baliunas et a1. 1979). 
A suggestion due originally to Wilson (1960), that Ly- $\alpha$ radiation pres sure can drive mass loss, was recently investigated by Haisch, Linsky, and Basri (1979) in the context of the K2 IIIp star a Boo. They calculate a Ly- $\alpha$ flux based on a sophisticated radiative transfer solution, but use the optically-thin approximation for the force calculation and neglect velocity gradients in the transfer. Their results show that Ly $-\alpha$ pressure may be larger than gravity over a small volume, but that the total momentum added is vastly insufficient to generate mass loss, so that Alfven waves are suggested as the driving mechanism. This mechanism is very sensitive to the ionization state of the gas. If the material is too cold, then Ly- $\alpha$ is so optically thick that the force is negligible. If the gas is too hot, insufficient neutral hydrogen is available to absorb radiation. Since the temperature distribution adopted by Haisch et al. is not uniquely determined by the observations, it is unclear whether these flows actually become supersonic from Ly-a radiation pressure.

One attractive possibility, suggested by observations of strong turbulence in supergiant atmospheres and chromospheres, is that the mechanical energy in various forms of the turbulence can be deposited in such a way .as to drive mass loss. Hartmann and MacGregor (1979) have investigated the behavior of acoustic and magnetic wave modes as a function of stellar gravity for surface wave fluxes comparable to solar values. These waves are in principle more effective than radiation pressure in Ly- $\chi$ which represents only a fraction of the mechanical energy fluxes available for driving winds. Wave modes other than light carry a factor $\mathrm{v} / \mathrm{c}$ more momentum than light for a given energy flux, where $v$ is the mode speed. This factor is typically $\sim 10^{4}$ for sound and magnetic waves, so that the potential for driving mass loss with such waves is considerably greater than for photons.

The calculations show that while shocks can account for chromospheric heating, their dissipation lengths are generally too short to be effective in initiating outflow. In Fig. 6 we show some static chromospheric calculations for representative wave periods. The temperature rise in units of pressure scale heights is more gradual in low gravity stars, although these waves do not explicitly account for the transition-region corona interface. A suggestive feature of these results is indicated in the temperature structures calculated for longer-period waves with larger dissipation lengths. In dwarfs, increased dissipationwith height causes an increase in local gas temperature, but the opposite occurs at super- 


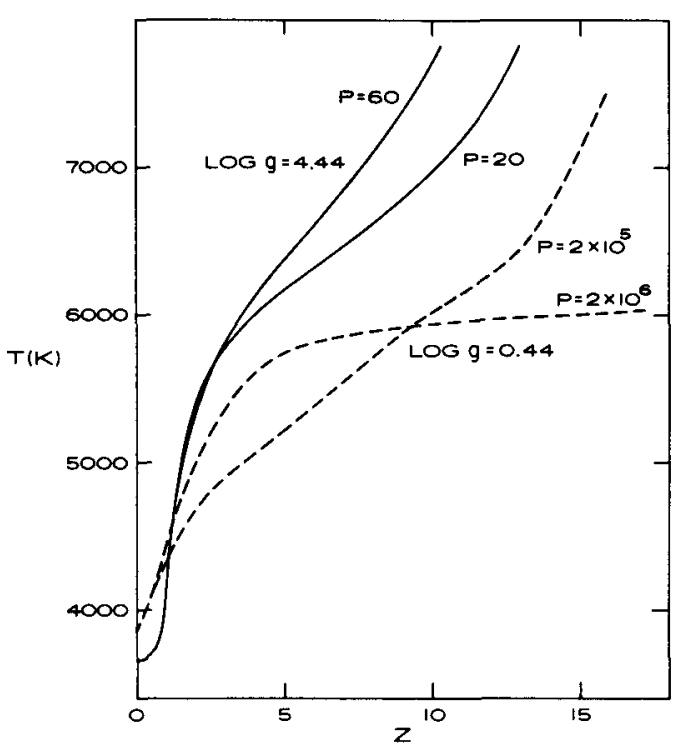

Figure 6 - Chromospheric temperature structures for dwarf (solid line) supergiant (broken line) gravities as a function of distance above the stellar surface, $z$, in units of base pressure scale heights. Increasing the period, $P\left(\mathrm{~s}^{-1}\right)$, of the acoustic waves heating the chromosphere acts to raise the upper chromospheric temperature in dwarfs. The opposite occurs in supergiants, as the waves tend to extend the density distribution. (From Hartmann and MacGregor 1979).

giant gravities. The reason is that at low gravity the waves "push" rather than heat, and the density distribution becomes extended. Such a result is in general agreement with the suggestion of Linsky and Haisch (1979) that mass loss suppresses high-temperature gas. However, these calculations are only part of the story, for coronal formulation is not accounted for even for dwarfs. In addition, the acoustic waves do not cause mass loss, they just extend the atmosphere.

Alfven waves, on the other hand, are very efficient in driving mass loss because of their long dissipation lengths. If one assumes wave surface fluxes comparable to those adopted for solar wind models, it is possible to arrive at reasonable mass loss rates. However, such wave-driven winds generally result in very high-velocity winds, unless some damping occurs within a few stellar radii (Fig. 7). Ion-neutral damping may become very important in winds that are observational1y known to be substantially neutral. Magnetic theories are unsatisfying in general because of the lack of observational quantities available for test. However, mechanical 


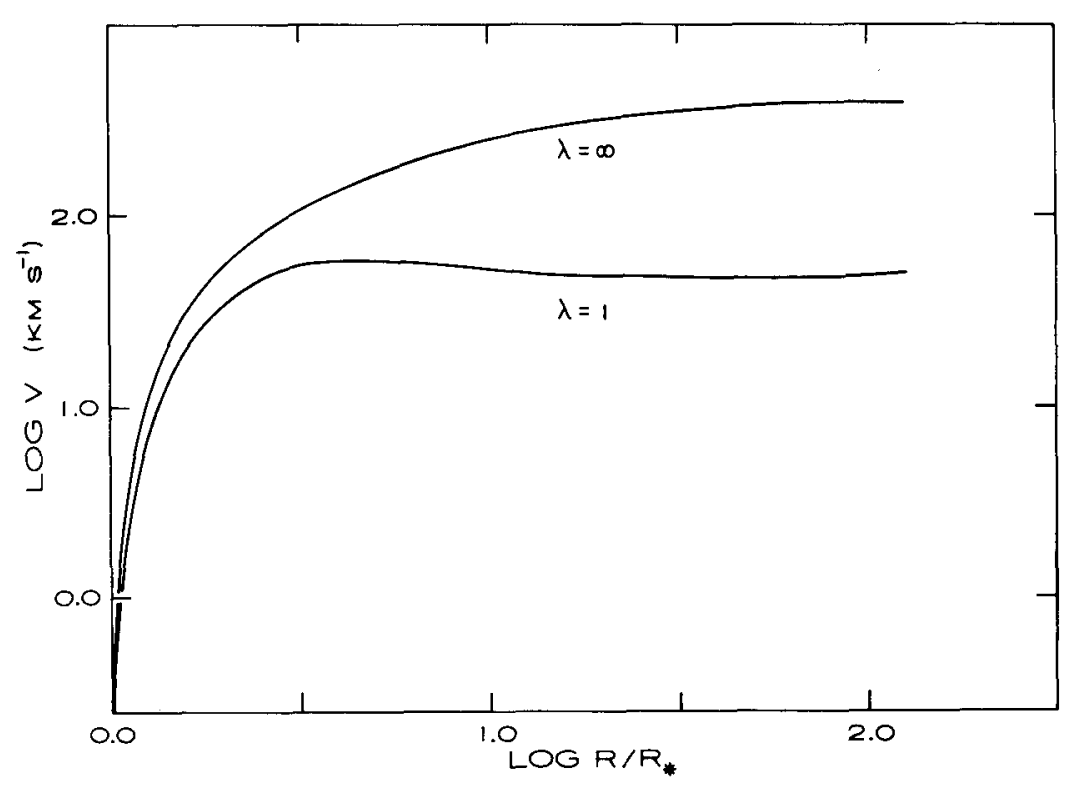

Figure 7 - Velocity structures of cool Alfven-wave driven winds for supergiants. Models in which the Alfven waves are undamped $(\lambda=\infty)$ have high terminal velocities. It is necessary to damp out the wave energy within $1 R_{*}$ of the surface in order to obtain terminal velocities of tens of $\mathrm{km} \mathrm{s}^{-1}$.

energy dissipation of the magnitude required to drive observed mass loss will necessarily result in heating of the wind, probably at larger distances than acoustic waves can be effective (Hartmann and MacGregor 1979). Therefore extended warm chromospheres seem to be a necessary feature of such wind models; this should be amenable to observational test, particularly with Zeta Aur systems.

We feel that recent observational evidence, such as that of hybrid chromospheres and winds, strengthens the case for a more unified picture of solar and stellar activity than has been presented in the past. Whether mass 10 s as well as chromospheric and coronal heating is a result of solar-type turbulence and activity operating in lower-gravity environments is a question that the new ultraviolet and $x$-ray observations, probing high-temperature atmospheric regions, may be able to answer.

This work is supported in part by NASA Grant NSG 5370 to the Harvard College observatory. 


\section{REFERENCES}

Baliunas, S.L., Avrett, E.H., Hartmann, L., and Dupree, A.K. 1979, Astrophys. Journ. Letters, in press.

Basri, G.B., and Linsky, J.L. 1979, Astrophys. Journ., in press.

Brown, A., Jordan, C., and Wilson, R. 1979, in Proc. Symp. The First Year of IUE, in press.

Carpenter, K.G., and Wing, R.F. 1979, Bu11. A.A.S., 11, 419.

Cassine11i, J.P. 1979, Ann. Rev. Astron. Astrophys., 17, 275.

Catura, R.C., Acton, L.W., and Johnson, H.M. 1975, Astrophys. Journ. Letters, 196, L 47 .

Deutsch, A.J. 1956, Astrophys. Journ., 123, 210.

Deutsch, A.J. 1960, in Stellar Atmospheres, ed. J.L. Greenstein (Chicago: Univ. of Chicago Press), p. 543.

Dupree, A.K. 1975, Astrophys. Journ. Letters, 200, L27.

Dupree, A.K. 1976, in Physique des Mouvements dans les Atmospheres Stellaires, ed. R. Cayrel and M. Steinberg, Editions du Centre National de la Recherche Scientifique, p. 439.

Dupree, A.K. 1980, in Highlights of Astronomy, Proc. of Joint Discussion of U1traviolet Astronomy, I.A.U. Gen. Assembly, in press.

Dupree, A.K., Black, J.H., Davis, R.J., Hartmann, L., and Raymond, J.C. 1979 , in Proc. Symp. The First Year of IUE, in press.

Durney, B.R.1973, in Stellar Chromospheres, ed. S.D. Jordan and E.H. Avrett, NASA SP-317, $\overline{\mathrm{p}}-282$.

Evans, R., Jordan, C., and Wilson, R. 1975, Mon. Not. Roy. Astron. Soc. $, 172,585$.

Gahm, G.F., Fredga, K., Liseau, R., and Dravins, D. 1979 , Astron. Astrophys., 73, L4.

Gehrz, R.D., and Woolf, N.J. 1971, Astrophys, Journ., 165, 285.

Haisch, B., Linsky, J.L., and Basri, G. 1979, preprint.

Hartmann, L., Davis, R.J., Dupree, A.K., Raymond, J.C., Schmidtke, P.C., and Wing, R.F. 1979a, Astrophys. Journ. Letters, in press.

Hartmann, L., Dupree, A. K., and Raymond, J.C. 1979b, in preparation.

Hartmann, L., and MacGregor, K. 1979, in preparation.

Hearn, A.G. 1975, Astron. Astrophys., 40, 355.

Kwok, S. 1975 , Astrophys. Journ., $198,583$.

Linsky, J.L. et a1., $197 \overline{8}$, Nature, $275,389$.

Linsky, J.L., and Haisch, B. 1979, Astrophys. Journ. Letters, 229, L27.

Mewe, R., Heise, J., Gronenschild, E.H.B.M., Brinkman, A.C., Schrijver, J., and den Boggende, A.J.F. 1975, Astrophys. Journ. Letters, 202, L67. Mulian, D.J. 1978, Astrophys. Journ., 226, 151.

Mullan, D., and Stencel, R. 1979, personal communication.

Nugent, J.' and Garmire, G. 1978, Astrophys. Journ. Letters, 226, L83.

Raymond, J.C., Noyes, R.W., and Stopa, M.P. 1979, Solar Phys., 61, 271.

Reimers, D. 1977, Astron. Astrophys., 57, 395.

Rosner, R. 1979 , personal communication.

Stencel, R.E. 1978, Astrophys. Journ. Letters, 223, L37.

Topka, K., Fabricant, D. Harnden, F.R., Jr., Gorenstein, P., and Rosner, R. 1979, Astrophys. Journ. Letters, 229, 661.

Vaiana, G., Forman, W., Giacconi, R., Gorenstein, P., Pye, J., Rosner, R., Seward, F., and Topka, K. 1979, Bul1. A.A.S., 11, 446.

Vitz, R.C., Weiser, H., Moos, H.W., Weinstein, A., and Warden, E.S. 1976, Astrophys. Journ. Letters, 205, L35.

Weymann, R. 1962, Astrophys. Journ., 136, 844.

Wilson, O.C. 1960 , Astrophys.Journ, $13 i, 75$.

Wing, R.F. 1978, in High Resolution Spectrometry (ed. M. Hack), Proceedings of the 4 th International Colloquium on Astrophysics, p. 683 . 